\title{
Modeling effect on the performance of impact and friction vibration absorbers
}

\author{
C. Mrad ${ }^{a}$, K. Chehaibi, A. Nasr and R. Nasri \\ National Engineering School of Tunis, PB 37, Le Belvedere 1002, Tunis, Tunisia
}

Received 10 June 2016, Accepted 1 August 2016

\begin{abstract}
Impact and friction vibration absorbers are introduced in mechanical systems to attenuate their vibrations with dissipating the vibratory energy through respectively collision or friction between both bodies. However, the contact between both bodies for both cases deserves to be investigated closely. This work aims firstly to study the effect of the collision modeling on the impact absorber efficiency. The impact is modeled under several forms: linear, semi-linear, and nonlinear; accordingly to elastic and viscous behaviors. The modeling, then the formulation, then the resolution of the problem are conducted for each case. The forced responses of the system are obtained, and a comparison is made to conclude on the absorber performance. This work aims secondly to examine the effect of friction modeling on the friction absorber efficiency. Two modeling approaches are considered: macroscopic and microscopic; accordingly to physical and tribological behaviors. The problem modeling is elaborated, followed by a formulation, then a resolution, to determine the forced responses of the system for different friction models. A comparison is made later to conclude on the friction absorber performance.
\end{abstract}

Key words: Mechanical vibration / impact absorber / friction absorber / modeling effect / absorbers performance

\section{Introduction}

The vibration attenuation of a mechanical system equipped with an impact absorber is due to the collisions between both bodies which cause energy dissipation [1]. The impacts are modeled using springs and dampers [2]. The contact between both bodies is to be investigated closely [3]. The most common impact models are based on stiffness and damping linearity and nonlinearity [4].

The most convenient collision modeling relies on the fact that the interaction forces act continuously during the impact. Therefore, the study can be done with only including the contact forces in the motion equations during the collision to reach a fair description of the system actual behavior $[5,6]$.

Three impact models are considered: Linear, semilinear, and nonlinear. The linear model is defined using a linear stiffness and a linear viscous damping, the linear stiffness becomes nonlinear for the semi-linear model (Hertz), and the linear viscous damping becomes nonlinear for the nonlinear model (Hunt-Crossley). For each impact model, using the continuous approach, an analytic study is conducted to determine the motion equations, it

\footnotetext{
${ }^{a}$ Corresponding author:

Charfeddine.Mrad@enit.rnu.tn
}

is followed by a numeric resolution to obtain the forced responses of the system equipped with the impact absorber. A comparison of the different impact models is made later to conclude on the impact absorber performance.

On the other hand, the vibration attenuation of a mechanical system equipped with a friction absorber is due to the friction between both bodies which causes energy dissipation [7]. Although that mass absorbers are widely used to reduce vibration levels [8], several studies showed that friction absorbers are more effective [9], but the contact between both bodies is to be investigated closely.

The recent modeling of friction proves the phenomenon complexity, some experimental observations show that friction changes with time [10]. The change is due to the surface quality, the material deformation, the temperature variation, the lubrication quality, and the accumulation of wear debris $[11,12]$.

Two modeling approaches are considered: macroscopic (physical) and microscopic (tribological). The macroscopic modeling is examined using kinetic friction (Coulomb), then kinetic and static frictions (Stribeck). The microscopic modeling is investigated using elastic contact (Dahl), then elastic and viscous contact (LuGre). For each friction model, an analytic study is elaborated to determine the motion equations, it is followed by a 


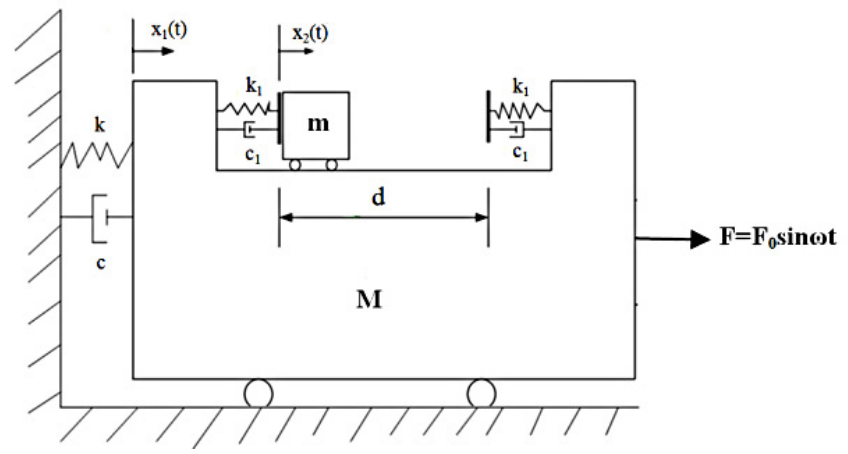

Fig. 1. System and Absorber, Linear collision.

numeric resolution to obtain the forced responses of the system equipped with the friction absorber. A comparison of the different friction models is made later to conclude on the friction absorber performance.

\section{Impact Absorber}

\subsection{Linear Modeling}

The mechanical system is of mass $M$, it is equipped with an impact absorber of mass $m$. During its vibration, the system collides with the absorber to cause vibratory energy dissipation. When the absorber mass hits the system mass, that is when the relative displacement of the absorber mass is less than the clearance width $(d)$ for the left impact or superior to the clearance width for the right impact, an impulsion force appears within the contact of both masses. For linear modeling, the impact is materialized by a linear spring $\left(k_{1}\right)$ for the elastic deformation, and a linear viscous damper $\left(c_{1}\right)$ for the energy dissipation. The system equipped with the absorber in forced vibration and with linear collision is presented in Figure 1. $k$ and $c$ denote respectively the stiffness and damping of the system, while $F$ refers to the excitation force of modulus $F_{0}$ and of pulsation $\omega . x_{1}$ and $x_{2}$ denote respectively the displacements of the system and the absorber masses.

The contact force is thus defined as:

$F_{c}=c_{1} \dot{y}+k_{1} y ; \quad y$ is the masses relative displacement

$$
\left(y=x_{2}-x_{1}\right) .
$$

The motion equations in forced vibration during the contact between both masses are therefore:

$$
\left\{\begin{array}{l}
M \frac{\mathrm{d}^{2} x_{1}}{\mathrm{~d} t^{2}}+c \frac{\mathrm{d} x_{1}}{\mathrm{~d} t}+k x_{1}=c_{1} \frac{\mathrm{d} y}{\mathrm{~d} t}+k_{1} y+F(t) \\
m \frac{\mathrm{d}^{2} x_{2}}{\mathrm{~d}^{2}}+c_{1} \frac{\mathrm{d} y}{\mathrm{~d} t}+k_{1} y=0
\end{array}\right.
$$

or:

$$
\left\{\begin{array}{l}
M \frac{\mathrm{d}^{2} x_{1}}{\mathrm{~d} t^{2}}+c \frac{\mathrm{d} x_{1}}{\mathrm{~d} t}+k x_{1}=c_{1} \frac{\mathrm{d} y}{\mathrm{~d} t}+k_{1} y+F(t) \\
m \frac{\mathrm{d}^{2}\left(x_{1}+y\right)}{\mathrm{d} t^{2}}+c_{1} \frac{\mathrm{d} y}{\mathrm{~d} t}+k_{1} y=0
\end{array}\right.
$$

Table 1. System-Absorber parameters, Linear collision.

\begin{tabular}{cc}
\hline Parameters & Values \\
\hline$M$ & $1 \mathrm{~kg}$ \\
$m$ & $0.4 \mathrm{~kg}$ \\
$k$ & $10^{3} \mathrm{~N} . \mathrm{m}^{-1}$ \\
$k_{1}$ & $400 \mathrm{~N} . \mathrm{m}^{-1}$ \\
$c$ & $0.1 \mathrm{Ns.m}{ }^{-1}$ \\
$c_{1}$ & $20 \mathrm{Ns.m}$ \\
$F_{0}$ & $2 \mathrm{~N}$ \\
$\omega$ & $47.4 \mathrm{rad} . \mathrm{s}^{-1}$ \\
\hline
\end{tabular}

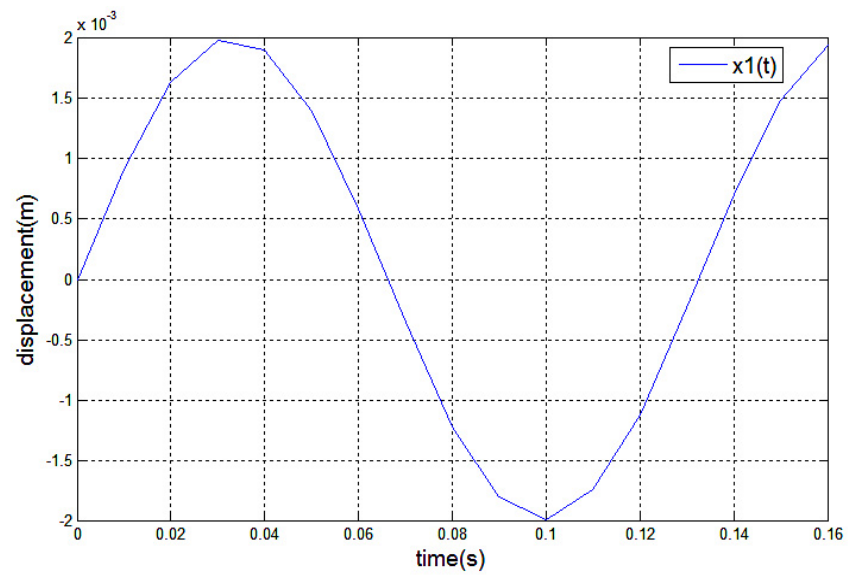

Fig. 2. System displacement, Linear collision.

The System-Absorber used parameters in linear collision are given in Table 1.

Figure 2 shows the obtained displacement of the system mass during the contact. The system is equipped with the absorber and the collision is linear.

The linear modeling presents three weaknesses [4]:

a. The impact force is discontinuous. In a realistic model, the two forces, elastic and viscous, start from zero and proceed progressively.

b. When the two masses split off, the masses relative velocity becomes negative, thus a negative force maintaining the two masses together appears.

c. The equivalent restitution coefficient does not depend on the impact velocity.

\subsection{Semi-Linear Modeling}

The collision is semi-linear, the contact between the system and the absorber masses is defined by a nonlinear stiffness and a linear viscous damping. The Hertz theory is used.

The system equipped with the absorber in forced vibration and with semi-linear collision is presented in Figure 3.

The contact force is thus defined as:

$F_{c}=k_{\mathrm{Hz}} y^{3 / 2} ; \quad y \quad$ is the masses relative displacement and $k_{\mathrm{Hz}}$ is the Hertz contact stiffness. 


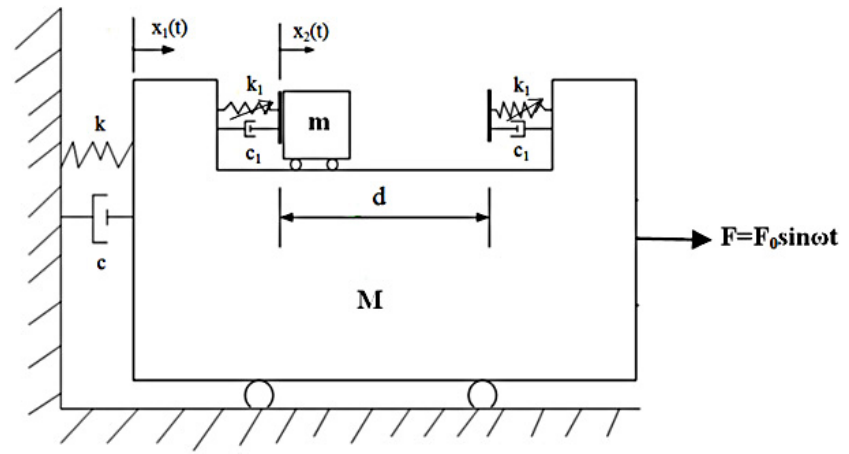

Fig. 3. System and Absorber, Semi-linear collision.

Some hypotheses are necessary to validate the contact force expression [6]:

a. The contact surface is small relatively to the masses geometries.

b. The collision occurs without friction.

c. The material is isotropic, elastic, and linear.

d. The contact time is enough long to establish a quasistatic state.

The contact stiffness is defined as:

$$
k_{\mathrm{Hz}}=\frac{4}{3 \pi} \frac{\sqrt{a}}{\left(1-\nu_{1}^{2}\right) / \pi E_{1}+\left(1-\nu_{2}^{2}\right) / \pi E_{2}}
$$

where, $a$ is the radius of the circle defining the contact surface:

$$
a=\sqrt[3]{\frac{3 N R}{4 E^{*}}} \text { with } \frac{1}{E^{*}}=\frac{1-\nu_{1}^{2}}{E_{1}}+\frac{1-\nu_{2}^{2}}{E_{2}}
$$

$E_{1}, E_{2}, \nu_{1}$ et $\nu_{2}$ are respectively the Young moduli and the Poisson coefficients of the system and the absorber masses; $N$ is the pressure force, normal to the contact, and $R$ is the radius of the Hertz sphere.

The motion equations in forced vibration during the contact between both masses are therefore:

$$
\left\{\begin{array}{l}
M \frac{\mathrm{d}^{2} x_{1}}{\mathrm{~d} t^{2}}+c \frac{\mathrm{d} x_{1}}{\mathrm{~d} t}+k x_{1}=c_{1} \frac{\mathrm{d} y}{\mathrm{~d} t}+k_{\mathrm{Hz}} y^{3 / 2}+F(t) \\
m \frac{\mathrm{d}^{2} x_{2}}{\mathrm{~d} t^{2}}+c_{1} \frac{\mathrm{d} y}{\mathrm{~d} t}+k_{\mathrm{Hz}} y^{3 / 2}=0
\end{array}\right.
$$

or:

$$
\left\{\begin{array}{l}
M \frac{\mathrm{d}^{2} x_{1}}{\mathrm{~d} t^{2}}+c \frac{\mathrm{d} x_{1}}{\mathrm{~d} t}+k x_{1}=c_{1} \frac{\mathrm{d} y}{\mathrm{~d} t}+k_{\mathrm{Hz}} y^{3 / 2}+F(t) \\
m \frac{\mathrm{d}^{2}\left(x_{1}+y\right)}{\mathrm{d} t^{2}}+c_{1} \frac{\mathrm{d} y}{\mathrm{~d} t}+k_{\mathrm{Hz}} y^{3 / 2}=0
\end{array}\right.
$$

The Hertz stiffness is to be determined wisely. Indeed, knowing the linear stiffness, it is possible to obtain the equivalent Hetz stiffness with equalizing the linear contact
Table 2. System-Absorber parameters, Semi-linear collision.

\begin{tabular}{cc}
\hline Parameters & Values \\
\hline$M$ & $1 \mathrm{~kg}$ \\
$m$ & $0.4 \mathrm{~kg}$ \\
$k$ & $10^{3} \mathrm{~N} . \mathrm{m}^{-1}$ \\
$k_{H z}$ & $3260 \mathrm{~N} \cdot \mathrm{m}^{-3 / 2}$ \\
$c$ & $0.1{\mathrm{Ns} . \mathrm{m}^{-1}}^{-1}$ \\
$c_{1}$ & $20{\mathrm{Ns} . \mathrm{m}^{-1}}$ \\
$F_{0}$ & $2 \mathrm{~N}$ \\
$\omega$ & $47.4 \mathrm{rad} . \mathrm{s}^{-1}$ \\
\hline
\end{tabular}

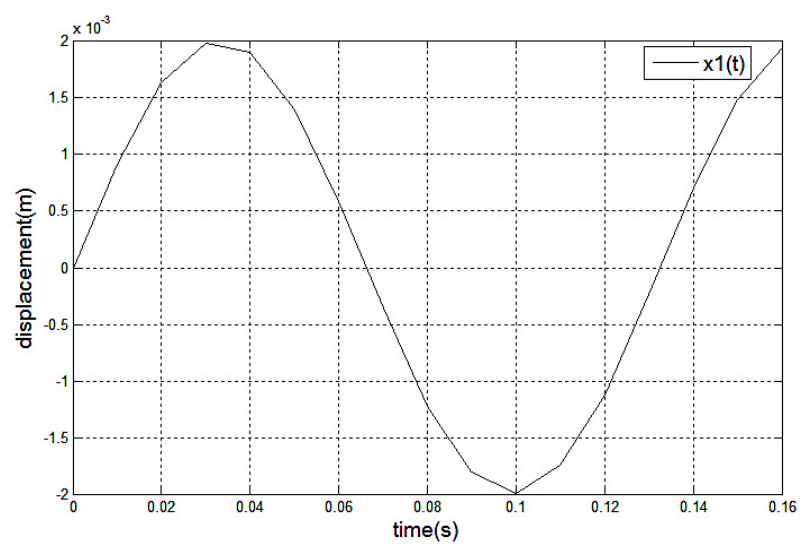

Fig. 4. System displacement, Semi-linear collision.

time and the Hertz contact time, defined respectively as follows:

$$
\begin{aligned}
T_{\text {Linear }} & =\frac{2 \pi m M}{\sqrt{m+M} \sqrt{4 m M k_{1}-c_{1}^{2}(m+M)}} \\
T_{\text {Hertz }} & =\frac{2.9432}{\dot{y}_{0}^{0.2}}\left(\frac{5}{4 K^{*} K_{\mathrm{Hz}}}\right)^{2 / 5} \text { with }:\left\{\begin{array}{l}
K *=\frac{\mathrm{m}+\mathrm{M}}{\mathrm{mM}} \\
\dot{y}_{0}=\dot{y}(0)
\end{array}\right.
\end{aligned}
$$

The System-Absorber used parameters in semi-linear collision are given in Table 2 .

Figure 4 shows the obtained displacement of the system mass during the contact. The system is equipped with the absorber and the collision is semi-linear.

The semi-linear modeling presents three weaknesses [5]:

a. It is only valid for elastic deformation

b. It does not cover nonlinear energy dissipation

c. It is only valid for low collision velocities

\subsection{Nonlinear Modeling}

The linear viscous damping does not reflect accurately the impact energy dissipation. Therefore, Hunt and Crossley developed a contact model adding to Hertz elastic force a nonlinear damping force defined using a contact penetration.

The system equipped with the absorber in forced vibration and with nonlinear collision is presented in Figure 5 . 


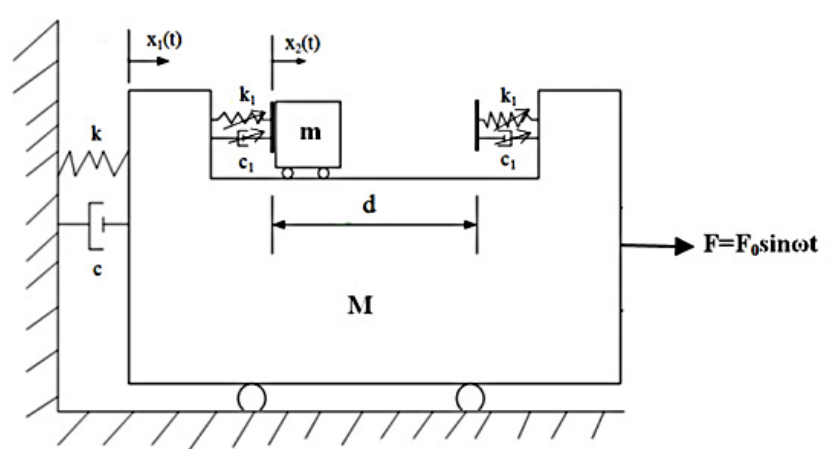

Fig. 5. System and Absorber, Nonlinear collision

The Hunt-Crossly contact force is defined as:

$$
F_{c}=k_{\mathrm{Hz}} y^{3 / 2}+c_{1} y^{3 / 2} \dot{y}
$$

where:

$y$ is the masses relative displacement.

$\dot{y}$ is the masses relative velocity (penetration velocity).

$k_{\mathrm{Hz}}$ is the Hertz contact stiffness.

$c_{1}$ is the Hunt-Crosseley damping coefficient.

The motion equations in forced vibration during the contact between both masses are therefore:

$$
\left\{\begin{array}{l}
M \frac{\mathrm{d}^{2} x_{1}}{\mathrm{~d} t^{2}}+c \frac{\mathrm{d} x_{1}}{\mathrm{~d} t}+k x_{1}=k_{\mathrm{Hz}} y^{3 / 2}+c_{1} y^{3 / 2} \frac{\mathrm{d} y}{\mathrm{~d} t}+F(t) \\
m \frac{\mathrm{d}^{2} x_{2}}{\mathrm{~d} t^{2}}+k_{\mathrm{Hz}} y^{3 / 2}+c_{1} y^{3 / 2} \frac{\mathrm{d} y}{\mathrm{~d} t}=0
\end{array}\right.
$$

or:

$$
\left\{\begin{array}{l}
M \frac{\mathrm{d}^{2} x_{1}}{\mathrm{~d} t^{2}}+c \frac{\mathrm{d} x_{1}}{\mathrm{~d} t}+k x_{1}=k_{\mathrm{Hz}} y^{3 / 2}+c_{1} y^{3 / 2} \frac{\mathrm{d} y}{\mathrm{~d} t}+F(t) \\
m \frac{\mathrm{d}^{2}\left(x_{1}+y\right)}{\mathrm{d} t^{2}}+k_{\mathrm{Hz}} y^{3 / 2}+c_{1} y^{3 / 2} \frac{\mathrm{d} y}{\mathrm{~d} t}=0
\end{array}\right.
$$

The Hunt-Crossley damping is to be determined wisely. It is function of the Hertz stiffness, the restitution coefficient $(e)$, and the masses relative velocity. It is defined as follows:

$$
c_{1}=\frac{3 k_{\mathrm{Hz}}\left(1-e^{2}\right)}{4 \dot{y}}
$$

The System-Absorber used parameters in nonlinear collision are given in Table 3 .

Figure 6 shows the obtained displacement of the system mass during the contact. The system is equipped with the absorber and the collision is nonlinear.

\subsection{Comparison}

The collision linear model is not too accurate. However, it is often used due to its simplicity. It takes into account linearly and respectively the elastic deformation and the energy dissipation, but does not cover plastic deformation.
Table 3. System-Absorber parameters, Nonlinear collision.

\begin{tabular}{cc}
\hline Parameters & Values \\
\hline$M$ & $1 \mathrm{~kg}$ \\
$m$ & $0.4 \mathrm{~kg}$ \\
$k$ & $10^{3} \mathrm{~N} . \mathrm{m}^{-1}$ \\
$k_{\mathrm{Hz}}$ & $3260{\mathrm{~N} . \mathrm{m}^{-3 / 2}}^{-1}$ \\
$c$ & $0.1{\mathrm{Ns} . \mathrm{m}^{-1}}^{-3 / 2}$ \\
$c_{1}$ & $469.5{\mathrm{Ns} . \mathrm{m}^{-3}}$ \\
$F_{0}$ & $2 \mathrm{~N}$ \\
$\omega$ & $47.4{\mathrm{rad} . \mathrm{s}^{-1}}$
\end{tabular}

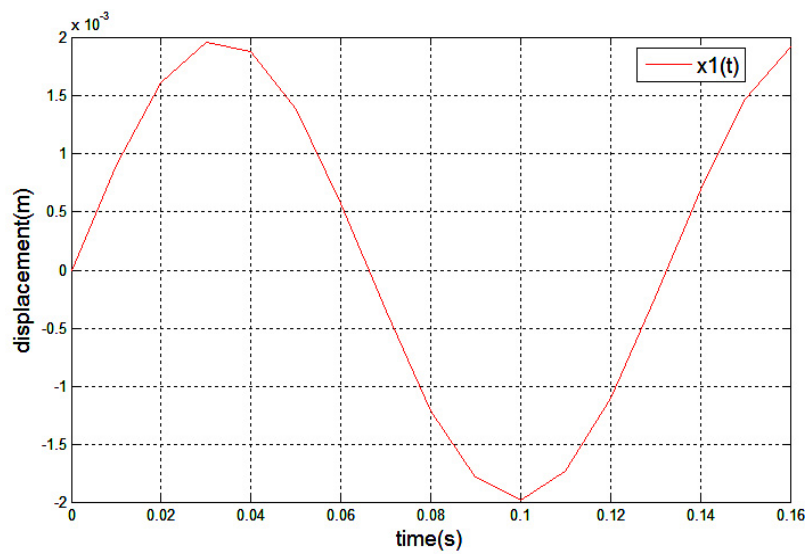

Fig. 6. System displacement, Nonlinear collision.

The collision semi-linear model is more accurate. It takes into account the nonlinearity of the elastic deformation, but does not cover plastic deformation and nonlinear energy dissipation, and is only valid for low collision velocities. Besides, it presents the same disadvantages of Hertz contact: the contact surface is small relatively to the masses, the contact surface is perfectly smooth, the material is isotropic, elastic, and linear, and the contact time is enough long. Therefore, the semi-linear model is to be used cautiously, especially when the material has high elastic deformation.

The collision nonlinear model is further accurate. It is based on the Hunt-Crossley contact which adds nonlinear damping to the Hertz contact. It takes into account nonlinearly and respectively the elastic deformation and the energy dissipation. Besides, the damping depends on the deformation, which means that the contact surface may vary during the collision. In addition, the contact force does not present any discontinuity, it stars from zero and ends at zero.

The obtained system vibrations for the linear and semi-linear collisions are similar, while the system vibration for the nonlinear collision is close to the previous responses but is more attenuated, Figure 7 . 


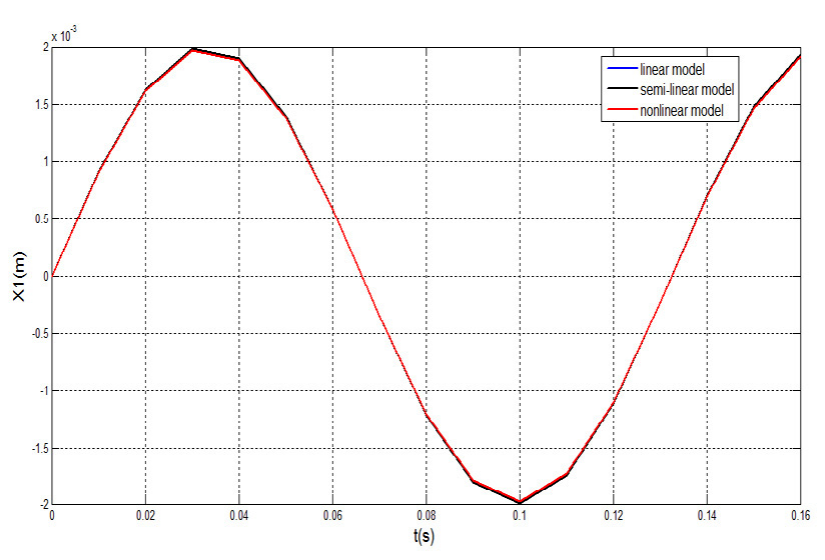

Fig. 7. System displacement, All collision models.

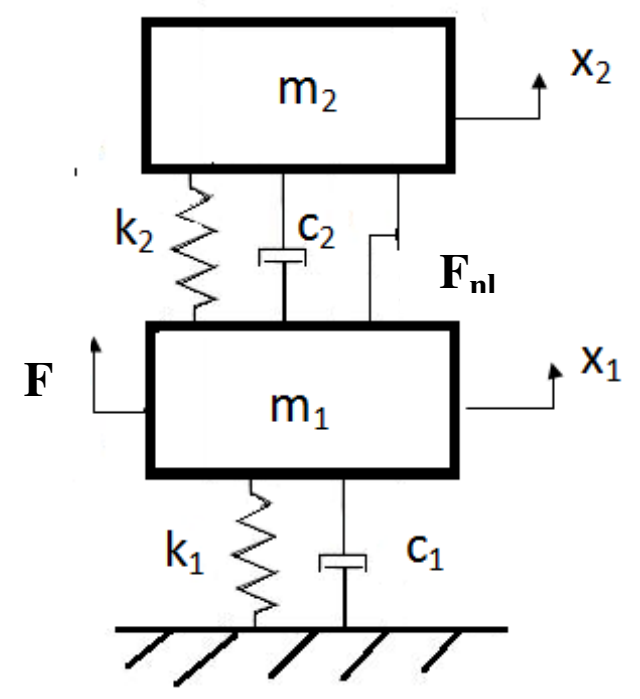

Fig. 8. System and Absorber.

\section{Friction absorber}

\subsection{System and absorber}

The mechanical system is of mass $m_{1}$, of stiffness $k_{1}$, and of damping constant $c_{1}$, the stiffness is linear and the damping is viscous. The friction absorber is of mass $m_{2}$, of stiffness $k_{2}$, and of damping constant $c_{2}$, the stiffness is linear and the damping is viscous. Friction between the two bodies is added, it is described by the force $F_{n l}$ (nonlinear), and may be materialized using different models. The system equipped with the absorber in forced vibration is presented in Figure 8, $F$ refers to the excitation force of modulus $F_{0}$ and of pulsation $\omega, x_{1}$ and $x_{2}$ denote respectively the displacements of the system and the absorber masses.

The motion equations in forced vibration taking into account friction are as follows:

$$
\begin{gathered}
\ddot{X}+\left(2 \xi_{2} \omega_{2}(1+\mu)+2 \xi_{1} \omega_{1}\right) \dot{X}+\left((1+\mu) \omega_{2}^{2}+\omega_{1}^{2}\right) X_{1}^{2} x \\
+2 \xi_{1} \omega_{1} \dot{x}+\omega=\frac{F}{m_{1}}-F_{n l} r \ddot{x}-2 \xi_{2} \omega_{2} \dot{X}-\omega_{2}^{2} X=\frac{F_{n l}}{m_{2}}
\end{gathered}
$$

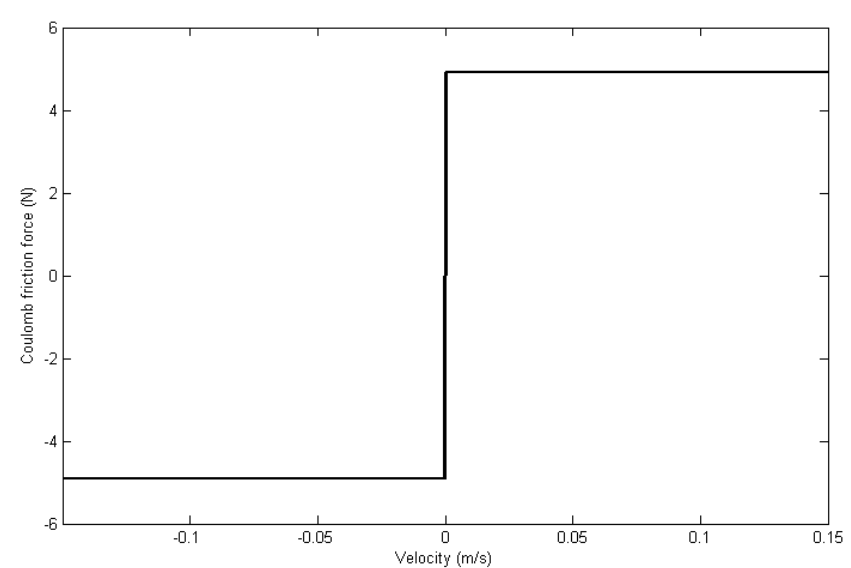

Fig. 9. Coulomb force.

where: $X=x_{1}-x_{2} ; x=x_{2} ; F=F_{0} \sin \omega t ; \omega_{1}=\sqrt{\frac{k_{1}}{m_{1}}}$; $\omega_{2}=\sqrt{\frac{k_{2}}{m_{2}}} ; \quad \xi_{1}=\frac{c_{1}}{2 m_{1} \omega_{1}} ; \xi_{2}=\frac{c_{2}}{2 m_{2} \omega_{2}} ; \mu=\frac{m_{2}}{m_{1}} ;$ and $r=\frac{1}{m_{1}}+\frac{1}{m_{2}}$

The friction force depends on the relative velocity sign. The diversity of friction models leads to investigate friction modeling. Two modeling approaches and four descriptive models are examined.

\subsection{Macroscopic modeling}

The friction macroscopic modeling is physical, it supposes an equivalent friction force acting within the contacting surfaces.

The Coulomb force takes into account kinetic friction, it is defined as (Fig. 9):

$$
F_{n l}=u_{c} N \operatorname{sign}(\dot{X})
$$

$u_{c}$ is the Coulomb kinetic friction coefficient, while $N$ is the normal force within the contacting surfaces.

The Stribeck force takes into account static friction in addition to kinetic friction, it is defined as (Fig. 10):

$$
F_{n l}=N\left(u_{c}+\left(u_{s}-u_{c}\right) e^{-\left(\frac{\dot{X}}{v_{s}}\right)^{2}}\right) \operatorname{sign}(\dot{X})
$$

$u_{s}$ is the Coulomb static friction coefficient, while $v_{s}$ is the Stribeck velocity.

The system vibratory behavior using Coulomb and Stribeck friction forces is investigated. The problem parameters are presented in Table 4, the system and the absorber are considered undamped.

Figures 11 shows the obtained displacement of the system mass for Coulomb friction, while Figure 12 shows the obtained displacement of the system mass for Stribeck friction, considering the excitation force.

For both frictions, the phenomenon stick-slip occurs. When the excitation force is weak, the absorber does not present any motion, the system and the absorber are reduced to one system. Besides, the responses of the system 


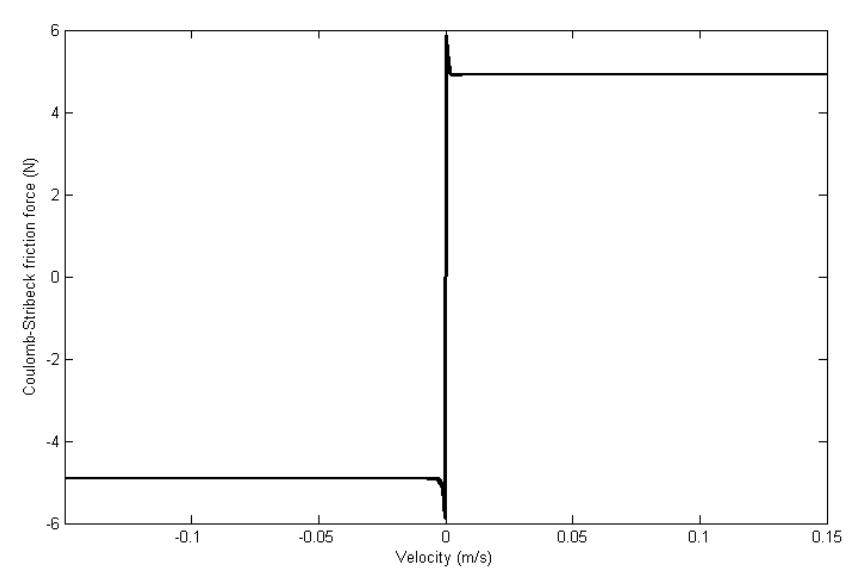

Fig. 10. Stribeck force.

Table 4. System-Absorber parameters, macroscopic friction.

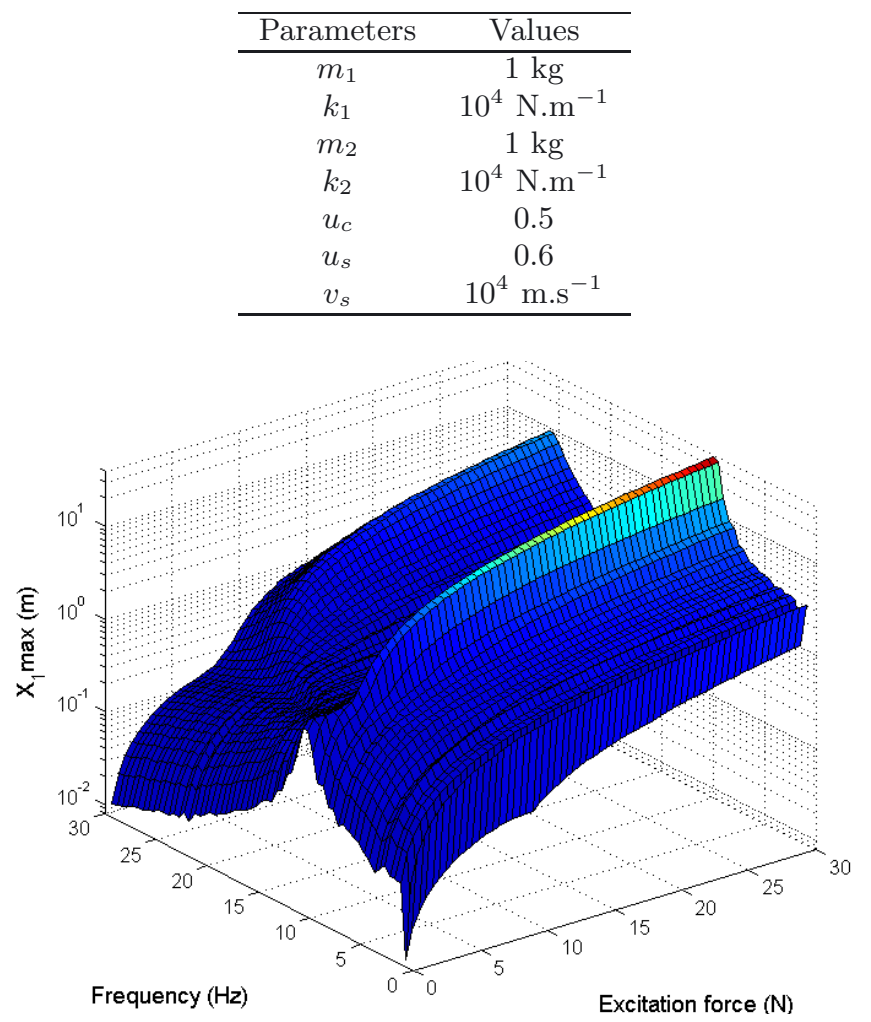

Fig. 11. System displacement-Coulomb.

equipped with the absorber are similar for both frictions, but the system vibration is more attenuated for Stribeck.

The shifting from the stick state to the slip state demands an enough strong excitation force to put the absorber into motion, thus to create an absorber acceleration force $\left(F_{a}\right)$ superior to the absorber friction force $\left(F_{n l}\right)$. The absorber acceleration force is defined as:

$$
F_{a}=m_{2} \ddot{X}
$$

Figure 13 shows the evolution of the absorber acceleration and friction forces ratio for Coulomb and Stribeck

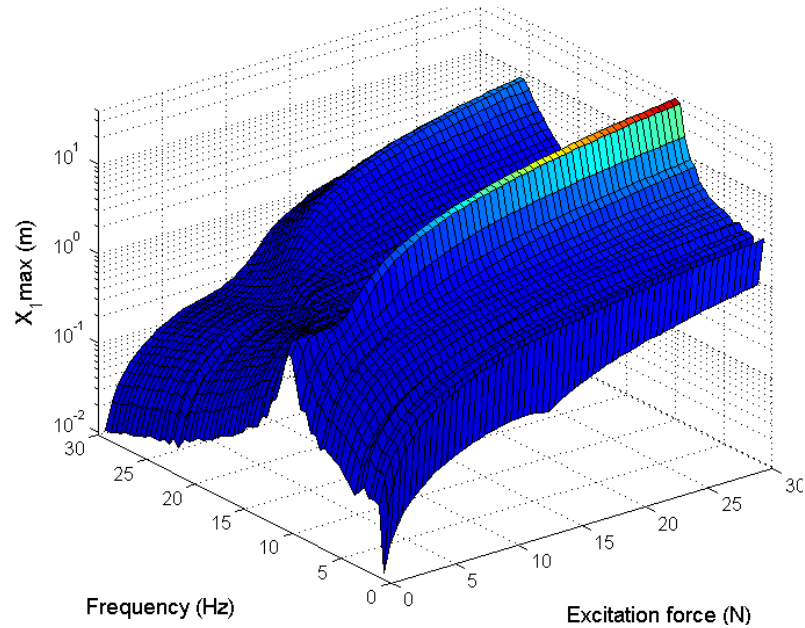

Fig. 12. System displacement-Stribec.

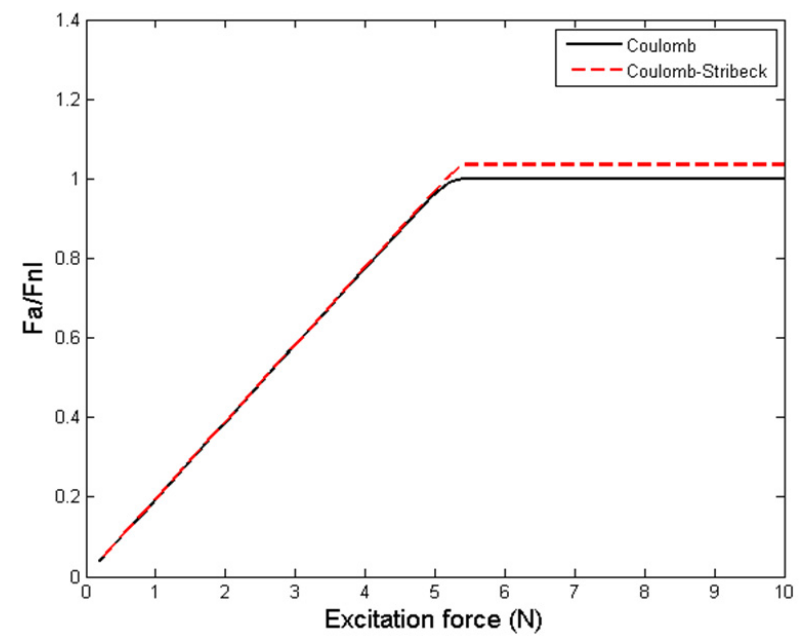

Fig. 13. Forces ratio, Coulomb-Stribeck.

frictions, whereas Figure 14 presents the evolution of the absorber displacement for both frictions.

Three peculiar zones are present: jammed absorber $\left(F_{a}\right.$ inferior to $\left.F_{n l}\right)$, partially gliding absorber $\left(F_{a}\right.$ slightly superior to $\left.F_{n l}\right)$, and totally gliding absorber $\left(F_{a}\right.$ neatly superior to $\left.F_{n l}\right)$. The Stribeck model presents a less rapid takeoff relatively to the Coulomb model, but the two models are similar regarding the absorber displacement.

\subsection{Microscopic modeling}

The friction microscopic modeling is tribological, it supposes a thorough knowledge of the two contacting surfaces.

Dahl considers the junction points between the contacting surfaces as equivalent to an elastic linkage which breaks under tangential loading, he refers to the stressstrain chart of materials. The Dahl force is defined as (Fig. 15):

$$
\left\{\begin{array}{l}
F_{n l}=\sigma_{0} z \\
\dot{z}=\dot{X}-\frac{\sigma_{0}|\dot{X}|}{F_{c}} z
\end{array}\right.
$$




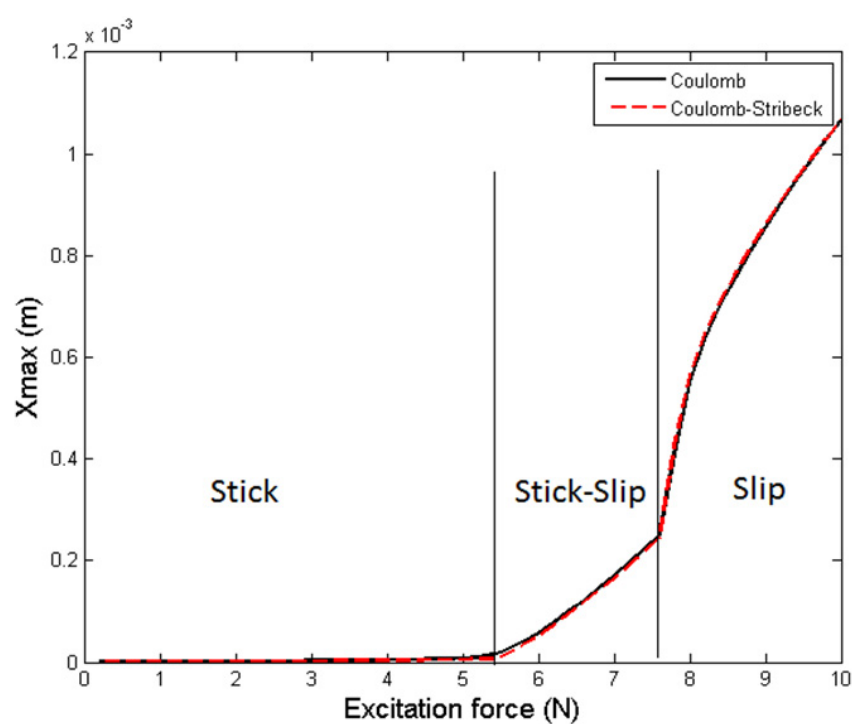

Fig. 14. Absorber displacement, Coulomb-Stribeck.

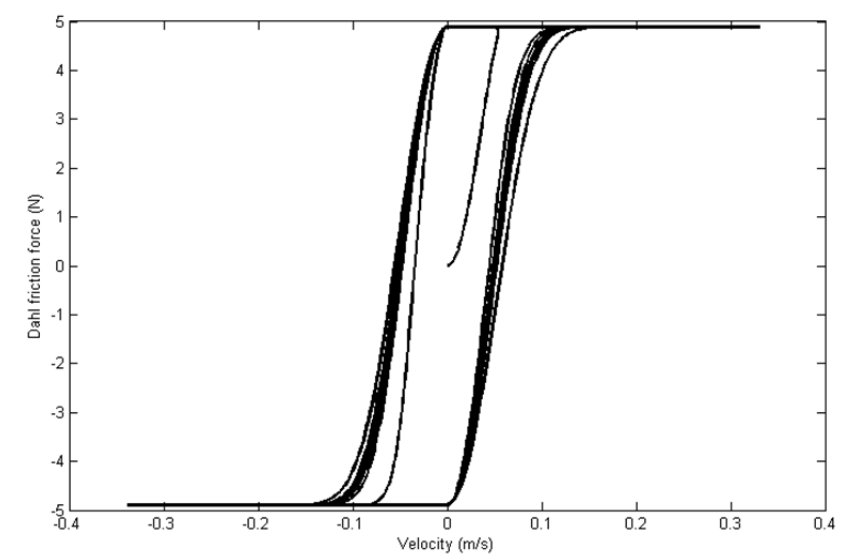

Fig. 15. Dahl force.

$z$ is the contact junction deflexion, while $\sigma_{0}$ is the contact junction stiffness, and $F_{c}$ is the Coulomb kinetic force.

LuGre added the contact junction damping, the Sribeck effect, and the lubrication effect. The LuGre force is defined as (Fig. 16):

$$
F_{n l}=\sigma_{0} z+\sigma_{1} \dot{z}+\sigma_{2} v
$$

where: $\dot{z}=\dot{X}-\frac{\sigma_{0}|\dot{X}|}{g(\dot{X})} z ; g(\dot{X})=F_{c}+\left(F_{s}-F_{c}\right) e^{-\left(\frac{\dot{X}}{v_{s}}\right)^{2}} ;$ and $v=\dot{X}$

$\sigma_{0}, \sigma_{1}$, and $\sigma_{2}$ denote respectively the contact junction stiffness, the contact junction damping, and the lubrication damping

The system vibratory behavior using Dahl and LuGre friction forces is investigated. The problem parameters are presented in Table 5, the system and the absorber are considered undamped and the lubrication damping is omitted.

Figure 17 shows the obtained displacement of the system mass for Dahl friction, while Figure 18 shows the

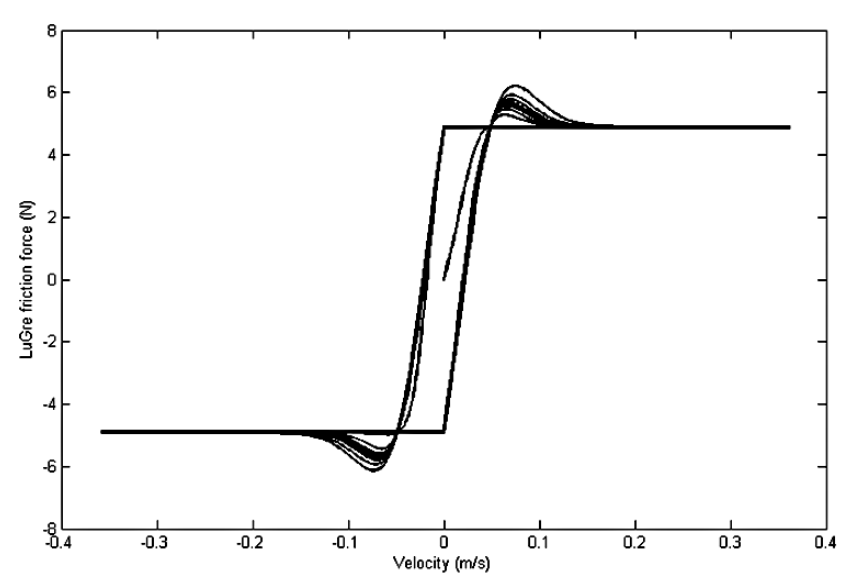

Fig. 16. LuGre force.

Table 5. System-Absorber parameters, microscopic friction.

\begin{tabular}{cc}
\hline Parameters & Values \\
\hline$m_{1}$ & $1 \mathrm{~kg}$ \\
$k_{1}$ & $10^{4} \mathrm{~N} \cdot \mathrm{m}^{-1}$ \\
$m_{2}$ & $1 \mathrm{~kg}$ \\
$k_{2}$ & $10^{4} \mathrm{~N} \cdot \mathrm{m}^{-1}$ \\
$u_{c}$ & 0.5 \\
$u_{s}$ & 0.6 \\
$v_{s}$ & $10^{4} \mathrm{~m} . \mathrm{s}^{-1}$ \\
$\sigma_{0}$ & $10^{4} \mathrm{~N} \cdot \mathrm{m}^{-1}$ \\
$\sigma_{1}$ & $10^{2} \mathrm{Ns} \cdot \mathrm{m}^{-1}$ \\
\hline
\end{tabular}

obtained displacement of the system mass for LuGre friction, considering the excitation force.

For both models, the phenomenon stick-slip happens. When the excitation force is weak, the absorber does not present any motion, thus the system and the absorber become one system. Besides, the responses of the system equipped with the absorber are similar for both frictions, but the system vibration is more attenuated for LuGre.

Figure 19 shows the evolution of the absorber acceleration and friction forces ratio for Dahl and LuGre frictions, whereas Figure 20 presents the evolution of the absorber displacement for both frictions.

Three distinctive zones are present: jammed absorber $\left(F_{a}\right.$ inferior to $\left.F_{n l}\right)$, partially gliding absorber $\left(F_{a}\right.$ slightly superior to $\left.F_{n l}\right)$, and totally gliding absorber $\left(F_{a}\right.$ neatly superior to $\left.F_{n l}\right)$. The LuGre model is less uniform than the Dahl model, although that the evolution of the absorber displacement is similar for both models.

\subsection{Comparison}

The friction macroscopic modeling supposes an equivalent friction force acting within the contacting surfaces. Two descriptive models are retained: Coulomb and Stribeck. The Coulomb model takes into account kinetic friction but does not consider static friction. The Stribeck model takes into account static friction in addition to kinetic friction. The macroscopic modeling does not consider contacting surfaces stiffness and damping. 


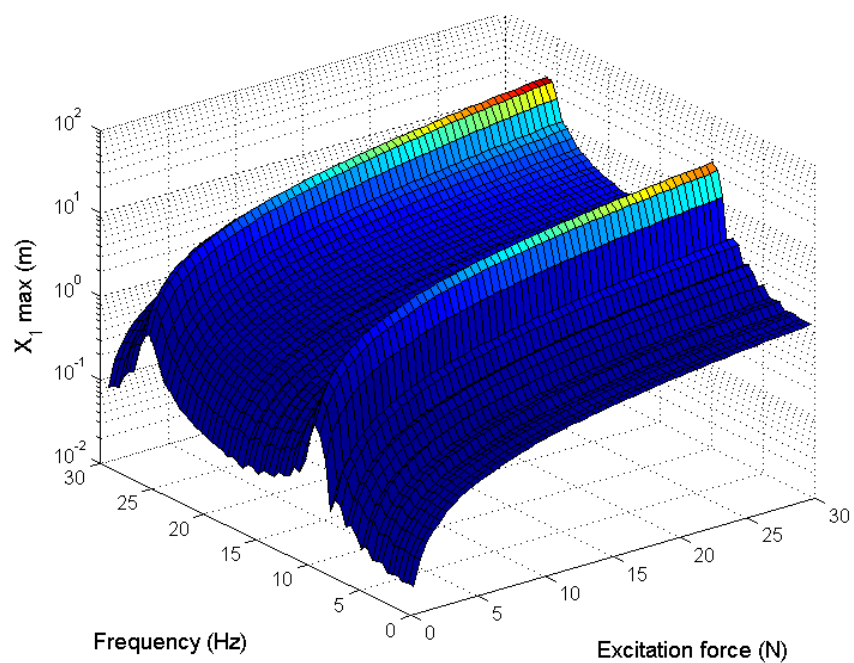

Fig. 17. System displacement-Dahl.

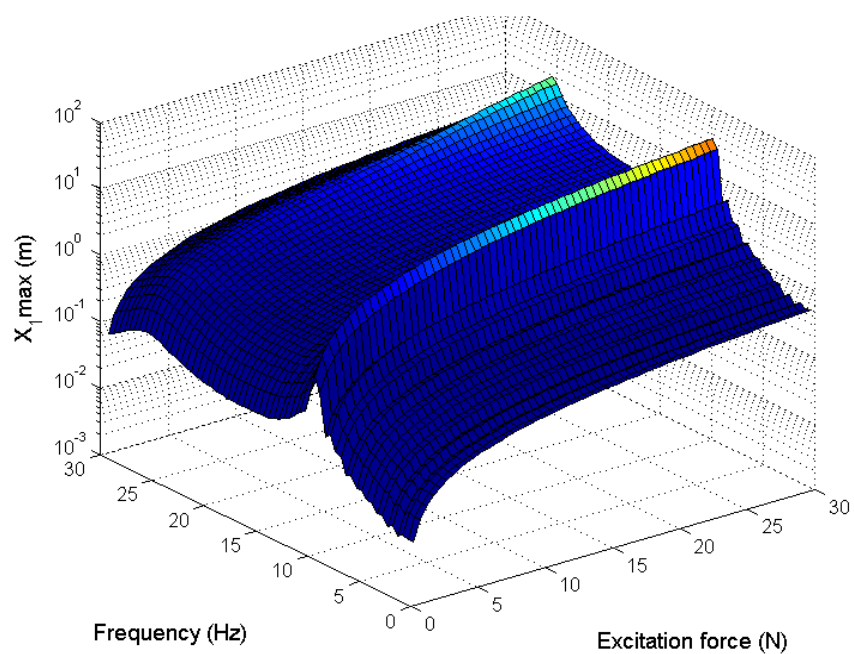

Fig. 18. System displacement-LuGre.

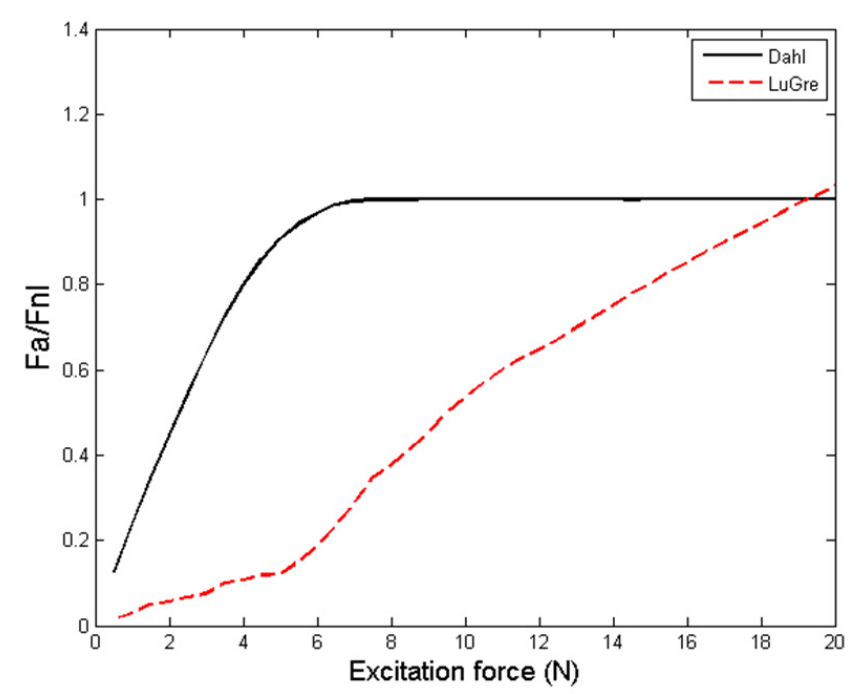

Fig. 19. Forces ratio, Dahl-LuGre.

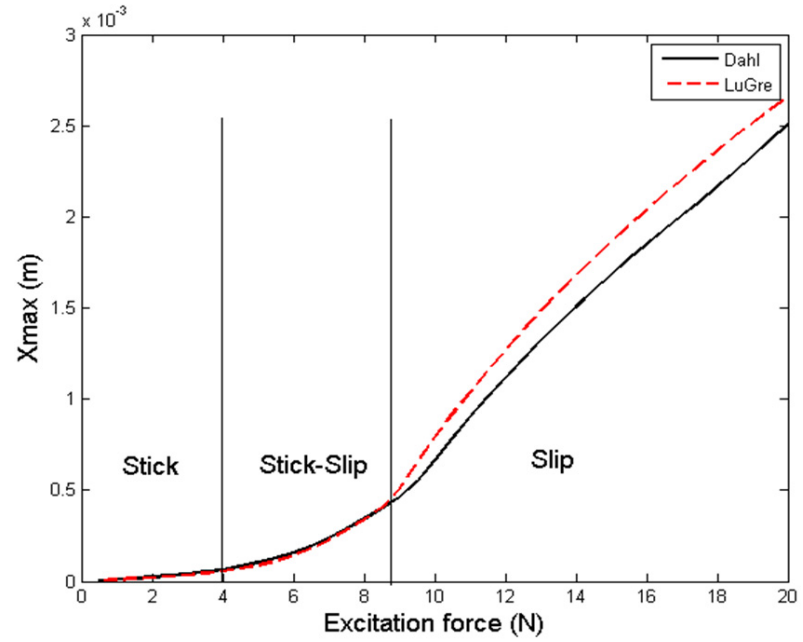

Fig. 20. Absorber displacement, Dahl-LuGre.

The Coulomb model is not too accurate, however it is often used due to its simplicity.

The friction microscopic modeling supposes a thorough knowledge of the contacting surfaces. Two descriptive models are retained: Dahl and Lugre. Dahl is based on the Coulomb model and takes into account contacting surfaces stiffness, but does not consider contacting surfaces damping. The LuGre model is based on the Stribeck model and takes into account the damping in addition to the stiffness of the contacting surfaces. The microscopic modeling considers both physical and tribological aspects of the contacting surfaces.

The results show that the Coulomb and Stribeck models are similar, although that the system vibration is more attenuated for Stribeck, apparently due to the increase of the friction force. Besides, the Dahl and LuGre models are also similar, but the system vibration is more attenuated for LuGre, apparently due to the presence of surfaces damping. However, the microscopic models present higher system vibrations comparatively to macroscopic models, apparently due to the considered surfaces stiffness.

\section{Conclusion}

Impact and friction vibration absorbers are introduced in mechanical systems to attenuate their vibrations with dissipating the vibratory energy through respectively collision or friction between both bodies. However, the contact between both bodies for both cases deserves to be investigated closely.

Regarding the impact absorber, three impact models are considered: Linear, semi-linear, and nonlinear. The linear model is defined using a linear stiffness and a linear viscous damping. the linear stiffness becomes nonlinear for the semi-linear model (Hertz), and the linear viscous damping becomes nonlinear for the nonlinear model (Hunt-Crossley). For each impact model, an analytic study is conducted to determine the motion equations, it is followed by a numeric resolution to obtain the 
forced responses of the system equipped with the impact absorber. A comparison of the different impact models is made later to conclude on the impact absorber performance. The results show that the impact models are similar although the accuracy difference, but the nonlinear model presents better vibration attenuation.

Regarding the friction absorber, two modeling approaches are considered: macroscopic and microscopic. The macroscopic modeling is examined using kinetic friction (Coulomb), then kinetic and static frictions (Stribeck). The microscopic modeling is investigated using elastic contact (Dahl), then elastic and viscous contact (LuGre). For each friction model, an analytic study is elaborated to determine the motion equations, it is followed by a numeric resolution to obtain the forced responses of the system equipped with the friction absorber. A comparison of the different friction models is made later to conclude on the friction absorber performance. The results show that the friction models are similar, but the microscopic models present lower vibration attenuation.

The contact between the mechanical system and the vibration absorber is to be considered cautiously, for both absorbers, to master any possible problems in practice.

Acknowledgements. This work is supported by the Tunisian ministry of higher education and scientific research, and is conducted within the system mechanics group (directed by Charfeddine MRAD) belonging to the Laboratory of Applied mechanics and engineering (National Engineering School of Tunis, University of Tunis el Manar, Tunisia).

\section{References}

[1] K.S. Marhadi, V.K. Kinra, Particle impact damping: effect of mass ratio, material, and shape, Department of Aerospace Engineering, Texas A\&M University, USA, 2004
[2] C.C. Cheng, J.Y. Wang, Free vibration analysis of a resilient impact damper, Int. J. Mech. Sci. 45 (2003) 589604

[3] J. Cheng, H. Xu, Inner mass impact damper for attenuating structure vibration, Int. J. Solids Struct. 43 (2006) $5355-5369$

[4] G. Gilardi, I. Sharf, Literature survey of contact dynamics modelling, Mech. Machine Theory 37 (2002) 12131239

[5] J.E. Jam, A.A.Fard, H.Taghavian, M. Khazaee, Acoustic behavior of single unit impact dampers, J. Curr. Res. Sci. 1 (2013) 351-355

[6] A. Afsharfard, A. Farshidianfar, An efficient method to solve the strongly coupled nonlinear differential equations of impact dampers, Archive Appl. Mech. 82 (2011) 977

[7] M.A. Lourozaa, N. Roitmanb, C. Maglutab, Vibration Reduction Using Passive Absorption System With Coulomb Damping, Mech. Systems Signal Process. 19 (2005) 537-549

[8] K. Liu, G. Coppola, Optimal design of damped dynamic vibration absorber for damped primary systems, Trans. Canadian Society Mech. Eng. 34 (2010) 1

[9] G.J. Stein, On dry friction modeling and simulation in kinematically excited oscillatory systems, J. Sound Vib. 311 (2008) 74-96

[10] H. Olsson, Friction models and friction compensation, Euro. J. Control 4 (1998) 176-195

[11] E.J. Berger, Friction modeling for dynamic system simulation, Appl. Mech. Rev. 55 (2002) 535-577

[12] J. Awrejcewicz, P. Olejnik, Analysis of dynamic systems with various friction laws, Appl. Mech. Rev. 58 (2005) 389-411 